\title{
Comparison of patients with acute ischemic stroke with and without COVID-19
}

\author{
Dilek Yılmaz Okuyan, Meltem Karacan Gölen \\ Konya Numune State Hospital, Selçuklu, Konya, Turkey
}

\begin{abstract}
Objectives: Information on the incidence of acute ischemic stroke (AIS), which is an important cause of morbidity and mortality, its association with COVID-19, and its course in infected patients in this pandemic period is limited. In this study, it was aimed to compare the demographic, clinical and stroke characteristics, and the laboratory, prognosis and mortality findings of patients with AIS with and without COVID-19. Methods: This study included 43 patients with a positive nasopharyngeal PCR test who were followed up for AIS, and 70 patients without COVID-19 who were followed up for AIS during the same period from the Konya Numune State Hospital, Turkey. Results: Poor prognosis and mortality were found to be significantly higher in the AIS group with COVID-19 compared with the non-COVID-19 AIS group. In the AIS group with COVID-19, a higher rate of intensive care unit (ICU) need (40\% vs. 5\%), higher intubation rate (35\% vs. 3\%) and longer hospital stay (12.9 \pm 10.8 vs. $6.6 \pm 4.0$ days) were observed. In the laboratory examinations, C-reactive protein, ferritin, D-dimer, troponin, and lactate dehydrogenase levels were found to be significantly higher in patients with AIS who were positive for COVID-19. An increase in D-dimer, ferritin and thrombocytopenia were found to be associated with mortality in the COVID-19 positive AIS group.

Conclusion: Patients with AIS and COVID-19 had a higher rate of ICU need, higher intubation rate, longer hospital stay, higher mortality, and poorer prognosis than those without COVID-19. High levels of ferritin, D-dimer and fibrin degradation products were associated with a poor prognosis.
\end{abstract}

Keywords: COVID-19, stroke, prognosis, mortality

\section{INTRODUCTION}

The coronavirus disease 2019 (COVID-19) pandemic caused by the new type of coronavirus 2 (SARS-CoV-2) was shown to affect both the respiratory tract and neurologic systems. The neurologic impairments that may occur as a result of the spread of the virus to the central nervous system through various mechanisms are headache, dizziness, impaired consciousness, seizures, encephalitis, cerebrovascular diseases, neuromuscular disorders, and peripheral nervous system involvement. Neurologic symptoms can occur in more than $50 \%$ of hospitalized patients. ${ }^{1}$

Acute ischemic stroke (AIS) is a lifethreatening complication of COVID-19. Information on the incidence of stroke, which is an important cause of morbidity and mortality, its association with COVID-19, and its course in infected patients in this pandemic period is increasing. Uncontrolled cytokine storm seen in severe disease can lead to multi-organ failure, and the activation of the microthrombotic pathway with destructive pathologic mechanisms mediated by the endothelial system can cause stroke. ${ }^{2}$

When assessing the link between COVID-19 and ischemic stroke, AIS has been increasingly documented in younger patients with COVID- $19^{3}$ or patients without cardiovascular risk factors and significant comorbidities. ${ }^{4}$ To date, the true incidence of AIS in patients with COVID-19 remains unclear, but in a study of 214 patients in Wuhan, the first epicenter of the pandemic, the incidence was $2.34 \% 5$, it was $0.9 \%$ in a study in New York ${ }^{6}$, and $1.2 \%$ in a more recent pooled analysis that evaluated 4,466 patients.

It is known that in the pathogenesis of SARS$\mathrm{CoV} 2$, the virus enters the cell by binding to the ACE-2 receptor. ACE-2 receptors, found in many tissues, are also present in endothelial cells. In the course of the disease, an increase in inflammatory markers, a deterioration in coagulation parameters such as elevation of 
D-dimer and thrombocytopenia, and an increase in blood pressure predispose to ischemic and hemorrhagic cerebrovascular events. ${ }^{8}$ It has been suggested that COVID-19 may induce a prothrombotic state accompanied by high fibrinogen and D-dimer levels. ${ }^{9}$ It has also been reported in various case series and reports that large vessel occlusion (LVO) is more common in patients with AIS and COVID-19. ${ }^{3}$

Given the rapidly increasing COVID-19 prevalence and its AIS complications globally, it is important to understand their relationship more deeply. The literature published to date has been limited to case reports, case series, and observational cohort studies. The number of studies including the effect of COVID-19 on mortality and prognosis in patients with AIS, and diagnostic and prognostic laboratory investigations are limited. In this study, it was aimed to compare the demographic, clinical, and stroke characteristics, neuroimaging findings, prognosis, and mortality of patients with AIS with and without COVID-19 admitted during the same period.

\section{METHODS}

This single-center retrospective study included 43 patients with a diagnosis of COVID-19 and AIS, and 70 patients with AIS without COVID-19 who were followed up in non-COVID-19 intensive care unit (ICU) between March 2020 and January 2021, from the 500 bed Konya Numune State Hospital, Konya, Turkey. All patients with COVID-19 in this study were diagnosed according to the World Health Organization (WHO) guidelines, and patients who were positive for SARS-CoV-2 in real-time reverse transcription-polymerase chain reaction (rRT-PCR) from nasopharyngeal swabs were included. All patients with AIS without COVID-19 were followed up in the nonCOVID-19 ICU after COVID-19 was excluded through clinical and laboratory findings and negative rRT-PCR tests from nasopharyngeal swabs.

Demographic characteristics, medical history, symptoms, clinical findings, laboratory findings, and imaging findings of both groups were evaluated retrospectively from electronic medical records. The diagnosis of AIS was confirmed with brain computed tomography (CT) and magnetic resonance imaging (MRI) findings and clinical symptoms. All neurologic symptoms were examined and approved by neurologists. Ischemic stroke types were classified according to the Trial of Org 10172 in Acute Stroke Treatment (TOAST) classification. ${ }^{10}$ Patients with AIS were divided into four main groups including total anterior circulation infarction (TACI), partial anterior circulation infarction (PACI), posterior circulation infarction (POCI), and lacunar infarction (LACI) according to the Bamford Clinical Classification recommended in the Oxfordshire Stroke Project (OCSP) study. ${ }^{11}$

The modified Rankin scale (MRS) and National Institutes of Health Stroke Scale (NIHSS) scores were recorded at the time of admission and 1 month after in all patients. MRS scores 3-6 were considered poor prognosis. In addition, 30-day mortality was evaluated in the patients.

Demographic data, risk factors, laboratory findings, clinical findings, prognosis, and mortality were compared between the groups.

Approval was obtained from the Local Ethics Committee (Protocol No: 2021-044) and the Ministry of Health for this retrospective study.

\section{Statistical analysis}

The normality of the distribution of continuous variables was tested using the Shapiro-Wilk test. Student's t-test (for normal data) or the MannWhitney U test (for non-normal data) were used to compare two independent groups. The Chisquare test was used to investigate the relationship between categorical variables. Receiver operating characteristics (ROC) curve analysis was performed to determine the diagnostic values of some numerical measurements.

Discrimination of the model was graded according to the area under the ROC curve, as follows: $0.5-0.6=$ fail discrimination, $0.6-0.7$ $=$ poor discrimination, $0.7-0.8=$ acceptable discrimination, $0.8-0.9=$ good discrimination, 0.9 $1=$ excellent discrimination. Statistical analysis was performed using the SPSS for Windows version 24.0 software and P-values $<0.05$ were accepted as statistically significant.

\section{RESULTS}

\section{Demographic and clinical characteristics}

In this study, 43 patients with AIS and COVID-19, and 70 patients with AIS without COVID-19 who were followed up during the same period were included. Of the patients included in the study, $67.3 \%$ (76/113) were male and 32.7\% (37/113) were female. The mean age was $70.56 \pm 11.02$ years in the AIS group with COVID-19 and 69.80 \pm 14.02 years in the non-COVID-19 AIS group.

When the risk factors and accompanying 
comorbidities of the patients were examined, it was observed that hypertension was the most common $(72 / 113,63.7 \%)$. Forty $(35.4 \%)$ patients had diabetes mellitus, 28 (24.7\%) had atrial fibrillation, $17(15 \%)$ had a history of stroke, $12(10.7 \%)$ were smokers, and six $(5.3 \%)$ had valvular heart disease. No significant difference was found between patients with AIS with and without COVID-19 in terms of demographic characteristics and risk factors (Table 1)

The mean NIHSS score at admission was $11.49 \pm 7.09$, and there was no significant

Table 1: Demographic and clinical features

\begin{tabular}{|c|c|c|c|c|}
\hline & Total $(n=113)$ & $\begin{array}{c}\text { Covid } 19 \\
\text { Positive AIS } \\
(n=43)\end{array}$ & $\begin{array}{c}\text { Covid } 19 \\
\text { Negative AIS } \\
(n=70)\end{array}$ & $\mathbf{P}$ \\
\hline Age $($ Mean \pm SD $)$ & $70.09 \pm 12.91$ & $70.56 \pm 11.02$ & $69.80 \pm 14.02$ & 0.763 \\
\hline \multirow[t]{2}{*}{ NIHSS at admission $($ Mean \pm SD) } & $11.49 \pm 7.09$ & $12.51 \pm 7.64$ & $10.87 \pm 6.71$ & 0.255 \\
\hline & $\mathrm{n}(\%)$ & $\mathrm{n}(\%)$ & $\mathrm{n}(\%)$ & \\
\hline Sex-Male & $76(67.3)$ & $33(76.7)$ & $43(61.4)$ & 0.092 \\
\hline Female & $37(32.7)$ & $10(23.3)$ & $27(38.6)$ & \\
\hline Hypertension & $72(63.7)$ & $28(65.1)$ & $44(62.9)$ & 0.808 \\
\hline Smoking & $12(10.7)$ & $6(13.9)$ & $6(8.6)$ & 0.367 \\
\hline Diabetes mellitus & $40(35.4)$ & $13(30.2)$ & $27(38.6)$ & 0.368 \\
\hline Hyperlipidemia & $43(38.1)$ & $14(32.6)$ & $29(41.4)$ & 0.346 \\
\hline Valvular heart disease & $6(5.3)$ & $2(4.7)$ & $4(5.7)$ & 0.807 \\
\hline Atrial Fibrillation & $28(24.7)$ & $8(18.6)$ & $20(27.1)$ & 0.233 \\
\hline Previus stroke & $17(15)$ & $5(11.6)$ & $12(17.1)$ & 0.426 \\
\hline Bamford Classification & & & & 0.101 \\
\hline $\begin{array}{l}\text { Total Anterior Circulation Infarcts } \\
\text { (TACI) }\end{array}$ & $28(24.7)$ & $10(23.2)$ & $18(25.7)$ & \\
\hline $\begin{array}{l}\text { Partial Anterior Circulation Infarcts } \\
\text { (PACI) }\end{array}$ & $29(25.7)$ & $15(34.9)$ & $14(20)$ & \\
\hline Posterior Circulation Infarcts (POCI) & $40(35.3)$ & $10(23.3)$ & $30(42.9)$ & \\
\hline Lacunar Infarcts & $16(14.2)$ & $8(18.6)$ & $8(11.4)$ & \\
\hline TOAST Classification & & & & 0.520 \\
\hline Large vessel occlusison (LVO) & $39(34.5)$ & $18(41.9)$ & $21(30)$ & \\
\hline Small vessel disease & $14(12.4)$ & $6(14)$ & $8(11.4)$ & \\
\hline Cardio-embolic & $35(31)$ & $12(27.9)$ & $23(32.9)$ & \\
\hline Stroke of Determined Origin & $2(1.8)$ & $0(0)$ & $2(2.9)$ & \\
\hline Stroke of Undetermined Origin & $23(20.4)$ & $7(16.3)$ & $16(22.9)$ & \\
\hline NIHSS & & & & 0.672 \\
\hline Mild (NIHSS 4 or less) & $22(19.5)$ & $8(18.6)$ & $14(20.0)$ & \\
\hline Moderate (NIHSS 5-10) & $39(34.5)$ & $13(30.2)$ & $26(37.1)$ & \\
\hline Severe (NIHSS > 10) & $52(46)$ & $22(51.2)$ & $30(42.9)$ & \\
\hline Poor outcome (mRS 3-6) & $55(48.7)$ & $27(62.8)$ & $28(40.0)$ & $0.019 *$ \\
\hline Mortality & $25(22.1)$ & $15(34.9)$ & $10(14.3)$ & $0.010 *$ \\
\hline
\end{tabular}

* NIHSS- National Institute of Heath Stroke Scale, mRS- Modified Rankin Score. TOAST, Trial of Org 10172 in Acute Stroke Treatment. Significant at 0.05 level; Chi-square test for categorical variables, Student t test for age, Mann whitney u test for NIHSS scores. 
difference between the AIS groups with and without COVID-19. According to the TOAST classification, LVO was found in $41.9 \%$ of the AIS group with COVID-19 and 30\% of the non-COVID-19 AIS group. Simultaneous multiple LVOs were detected in different vascular regions in $23.2 \%(10 / 43)$ of the AIS group with COVID-19, whereas this rate was $12.8 \%$ (9/70) in the non-COVID-19 AIS group $(\mathrm{p}=0.015)$. According to TOAST and Bamford classifications, no statistically significant difference was observed between the groups in terms of etiology and location (Table 1 and Figure 1).

Thirty-eight (88.3\%) of 43 patients with AIS and COVID-19 had findings of pneumonia in their lung CT. The median time from the first symptoms of COVID-19 to AIS was 10 (range, 1-20) days. Nine patients who were hospitalized for COVID-19 treatment developed AIS, and 34 patients were hospitalized due to the combination of AIS and COVID-19.

When the prognosis and mortality were evaluated, poor prognosis (mRS 3-6) and mortality were found to be significantly higher in the AIS group with COVID-19 compared with the nonCOVID-19 AIS group ( $\mathrm{p}=0.019$ and $\mathrm{p}=0.010$ ). In the AIS group with COVID-19, a higher rate of ICU need ( $40 \%$ vs. $5 \%$ ), higher intubation rate (35\% vs. $3 \%$ ), and longer hospitalization (12.9 \pm 10.8 vs. $6.6 \pm 4.0$ days) were observed.
In the AIS group with COVID-19, the poor prognosis rate was $62.8 \%$ and the mortality rate was $34.9 \%$.

The choice of treatment for ischemic stroke (antiaggregant/anticoagulant/intravenous thrombolytic therapy) was determined by the treatment team, which comprehensively reviewed the clinical syndrome, laboratory findings, and time of presentation. Four $(9.3 \%)$ patients in the AIS group with COVID-19 were administered intravenous thrombolytic therapy, and eight $(11.4 \%)$ patients in the non-COVID-19 AIS group received intravenous thrombolytic therapy.

\section{Laboratory findings}

When we evaluated the laboratory parameters, the number of lymphocytes, monocytes, and platelets in the AIS group with COVID-19 was found to be significantly lower than in the non-COVID-19 AIS group ( $\mathrm{p}=0.001, \mathrm{p}=0.041$, and $\mathrm{p}=0.002$, respectively). Lymphopenia was detected in $53.5 \%$ (23/43) of patients with COVID-19 and thrombocytopenia in $23.3 \%$ (10/43). C-reactive protein (CRP), ferritin, D-dimer, troponin, and lactate dehydrogenase (LDH) levels were found to be significantly higher in the AIS group with COVID-19 (Table 2).

When laboratory parameters affecting mortality were examined, a statistically significant

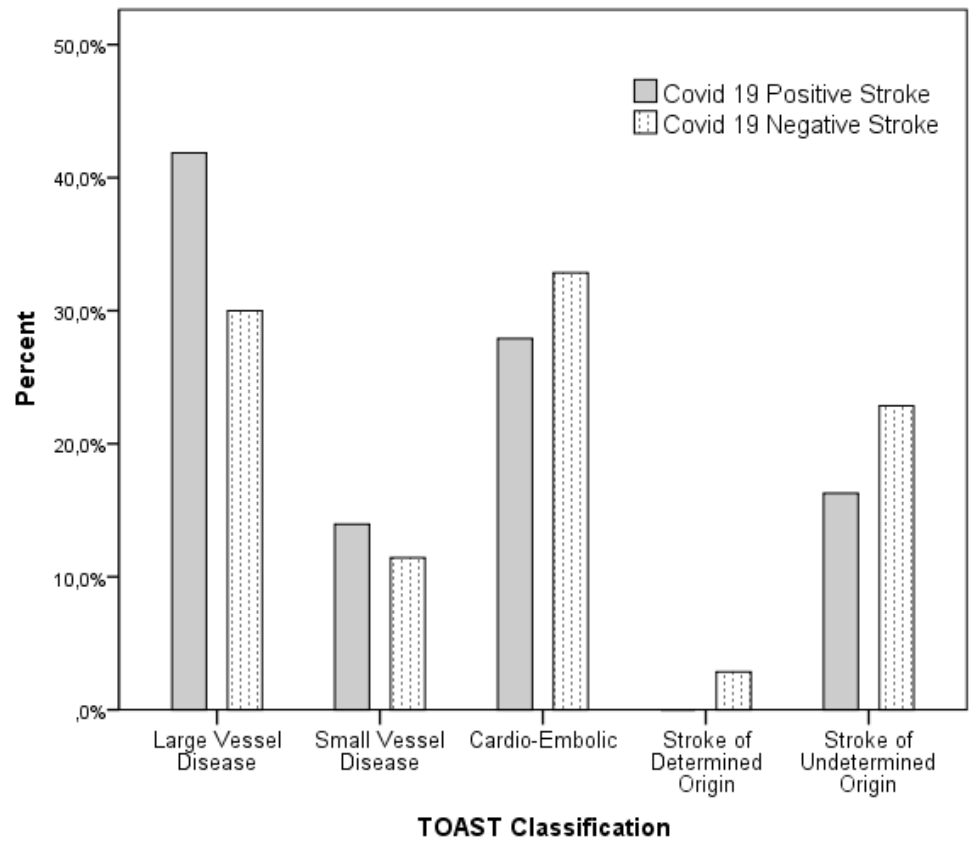

Figure 1. Distribution of etiologies in patients with AIS with and without COVID-19 according to TOAST classification 
Table 2: Comparison of laboratory findings of patients with AIS with and without COVID-19

\begin{tabular}{|c|c|c|c|c|}
\hline Laboratory Findings & Total $(n=113)$ & $\begin{array}{c}\text { Covid 19 } \\
\text { Positive AIS } \\
(\mathrm{n}=43)(\text { Median }) \\
\end{array}$ & $\begin{array}{c}\text { Covid } 19 \\
\text { Negative AIS } \\
(\mathrm{n}=70)(\text { Median}) \\
\end{array}$ & $\mathbf{P}$ \\
\hline White blood cell, $\times 10^{3} / \mathrm{uL}$ & $9.3[7-12.4]$ & $8.7[6.2-11.8]$ & $9.45[7.5-12.4]$ & 0.093 \\
\hline Neutrophil, $\times 10^{3} / \mathrm{uL}$ & $6.33[4.7-9]$ & $6.9[4.42-9.3]$ & $6.3[4.9-9]$ & 0.552 \\
\hline Lymphocyte , $\times 10^{3} / \mathrm{uL}$ & $1.43[0.9-2.35]$ & $0.9[0.63-1.43]$ & $1.9[1.3-2.54]$ & $0.001 *$ \\
\hline Monocyte, $\times 10^{3} / \mathrm{uL}$ & $0.67[0.46-0.8]$ & $0.49[0.4-0.95]$ & $0.7[0.5-0.8]$ & $0.041 *$ \\
\hline Platelet $\times 10^{3} / \mathrm{uL}$ & 255 [182-295] & 241 [155-309] & 257 [206-289] & $0.002 *$ \\
\hline $\mathrm{C}$ reactive protein $(\mathrm{CRP}), \mathrm{mg} / \mathrm{L}$ & $13[3.77-79]$ & $79.9[42-127]$ & $5.25[2.67-17.2]$ & $0.001 *$ \\
\hline Ferritin, $\mathrm{ng} / \mathrm{mL}$ & $122.5[39.5-373]$ & 364 [137.6-579] & $42.2[19.7-111]$ & $0.001 *$ \\
\hline Fibrinogen, mg/dL & $476.5[314-583]$ & 489 [424-583] & $341[293-750]$ & 0.343 \\
\hline D-dimer, $\mathrm{ng} / \mathrm{ml}$ & $520[185-2780]$ & $1195[430-6300]$ & $195[90-600]$ & $0.001 *$ \\
\hline Troponin, ng/mL & $10[4.6-34]$ & $22.45[8.1-77.8]$ & $7.8[3.9-15.9]$ & $0.001 *$ \\
\hline $\begin{array}{l}\text { Partial Thromboplastin } \\
\text { Time (APTT), s }\end{array}$ & $29.35[23.45-33]$ & 29 [24-34.1] & $29.4[23.3-31.6]$ & 0.552 \\
\hline $\begin{array}{l}\text { Lactate Dehydrogenase } \\
\text { (LDH), U/L }\end{array}$ & 324 [241-466] & $390.5[316-565]$ & $271[206-380.5]$ & $0.001 *$ \\
\hline Prothrombin time (PT), s & $13.2[12.1-14.4]$ & $13.5[12.4-15.6]$ & $13[12-13.8]$ & 0.236 \\
\hline Lymphopenia, $(\%)$ & $35(31)$ & $23(53.5)$ & $12(17.1)$ & $0.001 *$ \\
\hline Thrombocytopenia, (\%) & $13(11.5)$ & $10(23.3)$ & $3(4.3)$ & $0.002 *$ \\
\hline
\end{tabular}

*Significant at 0.05 level; Mann whitney u test for numerical variables. Median [25\%-75\%]

correlation was observed between the D-dimer, ferritin increase, and thrombocytopenia and mortality in the AIS group with COVID-19 $(\mathrm{p}=0.027, \mathrm{p}=0.034$, and $\mathrm{p}=0.015)$ (Table 3).

The values of the area under the ROC curve of the laboratory parameters were as follows: lymphocyte $0.772 \pm 0.05$, monocyte $0.615 \pm 0.06$, CRP $0.876 \pm 0.04$, ferritin $0.858 \pm 0.05$, D-dimer $0.806 \pm 0.05$, troponin $0.717 \pm 0.05$, and $\mathrm{LDH}$ $0.718 \pm 0.05$. The cut-off value of 36.8 for CRP was found to have a sensitivity of $76.74 \%$ and a specificity of $90.00 \% ; 111$ for ferritin was found to have a sensitivity of $83.72 \%$ and a specificity of $75.56 \%$; and 630 for D-dimer was found to have a sensitivity of $69.77 \%$ and a specificity of $77.78 \%$ (Table 4, Figure 3).

\section{DISCUSSION}

In this study, patients with AIS with and without COVID-19 followed up over a 10-month period were compared. The age range of patients with AIS and COVID-19 differs between studies. Although there were some reports in the literature about the coexistence of stroke in young patients with COVID-19, the mean age of patients with
COVID-19 in our study was $70.56 \pm 11.02$ years. Unlike case series in the literature, the number of patients aged under 50 years was two (4.6\%) in the AIS group with COVID-19 and five (7.1\%) in the non-COVID-19 AIS group, and no significant difference was found between the groups in terms of age. $3,12,13$

Vascular risk factors such as hypertension and diabetes, which may cause a more severe clinical picture in patients with COVID-19, also constitute additional risk factors for stroke. ${ }^{14}$ In a meta-analysis of six published studies evaluating 1,527 patients with COVID-19. common comorbid conditions including diabetes $(9.7 \%)$, cardiovascular disease $(16.4 \%)$, and hypertension (17.1\%) were reported. ${ }^{2}$ Although studies indicated that vascular risk factors and accompanying comorbidities were less common in patients with AIS and COVID-19 compared with those without COVID-19 ${ }^{15,16}$, there was no difference between the groups in our study regarding risk factors and comorbidities. Having similar demographic and risk factors in the control group provided an advantage for evaluating the effect of COVID-19 on the prognosis and mortality of ischemic stroke. 
Table 3: Mortality relationship with laboratory parameters in COVID-19-positive patients with AIS

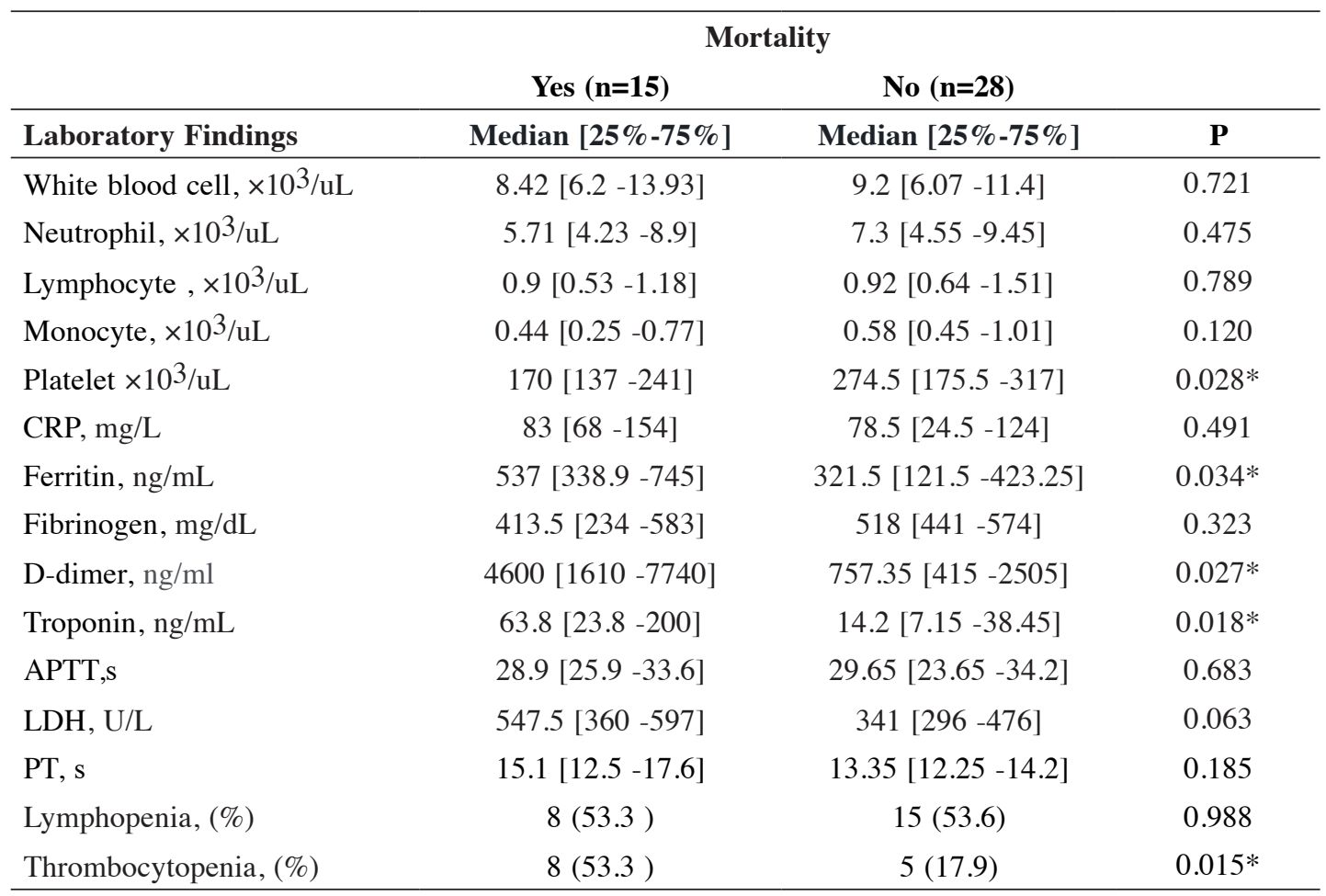

*CRP- C Reactive Protein, LDH- Lactate Dehydrogenase, APTT- Partial Thromboplastin Time, PT- Prothrombin time

Studies have reported conflicting data on the timing of thromboembolic complications in patients with COVID-19. ${ }^{17,18}$ Complications such as thrombosis, systemic angiopathy, and stroke are thought to occur in the advanced phase of the infection. In our study, the median duration from the first symptoms of COVID-19 to the development of AIS was 10 (range, 1-20) days. This finding was consistent with current assumptions that patients with severe COVID-19 may develop venous and arterial thromboembolism due to the prothrombotic state that develops following the early hyperinflammatory period of the cytokine storm. In a study in which more than 2,000 patients with COVID-19 were examined, it was found that the percentage of cerebrovascular events was higher in patients with severe disease (those in need of mechanical ventilation and basic life support), which could be demonstrated as evidence that stroke was seen more frequently in the second phase, when the severity of infection increased. ${ }^{19}$ However, it has been reported in case series that cerebrovascular events may occur in the early phase of the disease..$^{20,21}$

In previous studies, it was noted that in patients with COVID-19, moderate-severe AIS and LVO

Table 4: ROC curve analysis results for significant laboratory variables

\begin{tabular}{lccccccc}
\hline \multicolumn{1}{c}{ Variable } & AUC \pm SE & P & Cut-off & Sensitivity & 95\% CI & Specificity & 95\% CI \\
\hline Lymphocte & $0.772 \pm 0.05$ & 0.001 & $\leq 1.29$ & 74.42 & $58.8-86.5$ & 75.71 & $64.0-85.2$ \\
Monocyte & $0.615 \pm 0.06$ & 0.041 & $\leq 0.49$ & 51.16 & $35.5-66.7$ & 81.43 & $70.3-89.7$ \\
CRP & $0.876 \pm 0.04$ & 0.001 & $>36.8$ & 76.74 & $61.4-88.2$ & 90.00 & $80.5-95.9$ \\
Ferritin & $0.858 \pm 0.05$ & 0.001 & $>111$ & 83.72 & $69.3-93.2$ & 75.56 & $60.5-87.1$ \\
D-dimer & $0.806 \pm 0.05$ & 0.001 & $>630$ & 69.77 & $53.9-82.8$ & 77.78 & $60.8-89.9$ \\
Troponin & $0.717 \pm 0.05$ & 0.001 & $>20$ & 59.52 & $43.3-74.4$ & 78.26 & $66.7-87.3$ \\
LDH & $0.718 \pm 0.05$ & 0.001 & $>294$ & 80.95 & $65.9-91.4$ & 61.54 & $47.0-74.7$ \\
\hline
\end{tabular}

*CRP- C Reactive Protein, LDH- Lactate Dehydrogenase 


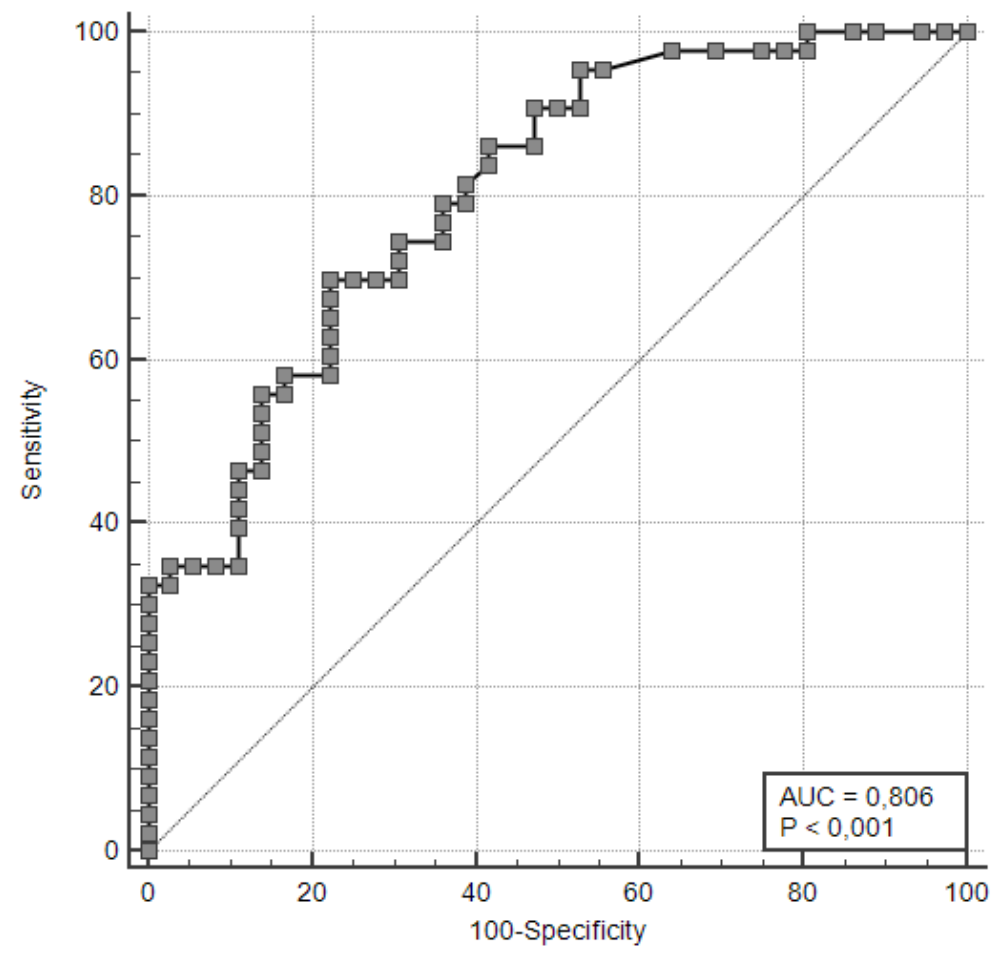

Figure 2. Receiver operating characteristics (ROC) curves of D-Dimer

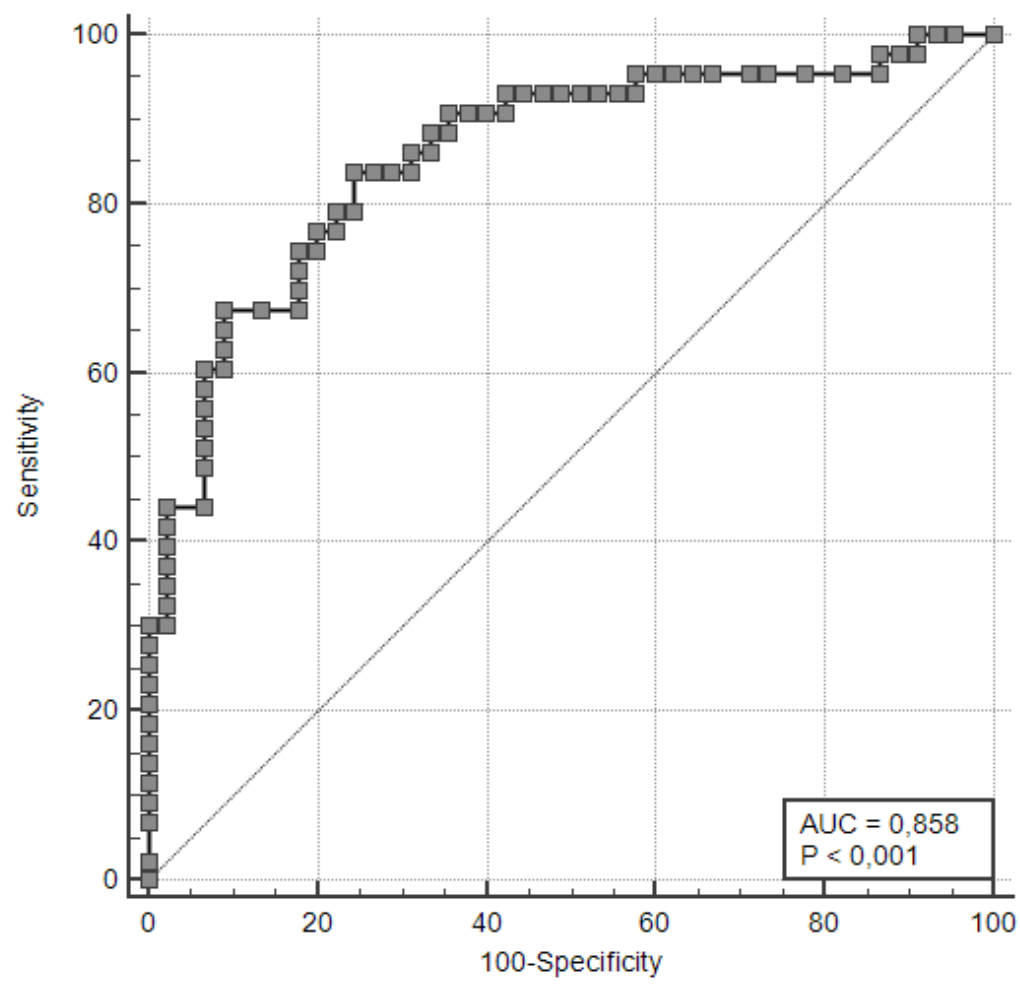

Figure 3. Receiver operating characteristics (ROC) curves of ferritin 
presented with large vessel thrombosis, embolism, stenosis or multiple vascular region patterns in imaging were observed more frequently. ${ }^{7}$ In our study, according to the TOAST classification, LVO was observed in $41.9 \%$ of the AIS group with COVID-19 and 30\% of the non-COVID-19 AIS group. In our study, NIHSS scores at admission in the COVID-19 AIS group were $12.51 \pm 7.64$ and $51.2 \%$ of the patients had severe NIHSS scores at the time of presentation. Although the interval between stroke symptom onset and admission was longer in the AIS group with COVID-19 than in the non-COVID-19 AIS group, no statistical significance was found between the groups. Simultaneous multiple LVO was detected in different vascular regions in $23.2 \%$ of patients with AIS and COVID-19 (10/43), whereas this rate was $12.8 \%$ in the non-COVID-19 AIS group (9/70). LVO and small vessel disease were observed more frequently in the COVID-19 AIS group, and cardioembolism was found to be higher in the non-COVID-19 AIS group. However, there was no significant difference between the groups in terms of location and etiology.

Of the patients with AIS and COVID-19, 88.3\% had evidence of pneumonia in lung CT. This finding was in accordance with results in the literature, that the incidence of ischemic stroke was higher in patients with severe COVID-19 findings. ${ }^{22,23}$ In our study, a higher rate of ICU need $(40 \%$ vs. $5 \%)$, higher intubation rate $(35 \%$ vs. $3 \%)$ and longer hospitalization ( $12.9 \pm 10.8$ vs. $6.6 \pm 4.0$ days) were observed in the AIS group with COVID-19. The rate of poor prognosis was $62.8 \%$, and the mortality rate was $34.9 \%$; both were significantly higher in the AIS group with COVID-19 than in the non-COVID-19 AIS group. Similar to our data, studies have shown that the presence of AIS is associated with higher mortality and worse prognosis in patients with COVID-19. ${ }^{24}$ Similar to our study, a study comparing patients with AIS without COVID-19, historical controls, and patients with AIS and COVID-19 showed that patients with concurrent COVID-19 had higher NIHSS scores, higher rates of atherosclerosis, and higher in-hospital mortality. ${ }^{6}$

It is very important to measure and evaluate laboratory markers of hemostasis disorders to better understand the pathogenesis of thrombosis. Patients with severe symptoms of COVID-19 may be at risk of thromboembolic events resulting from COVID-19-associated coagulopathy. Studies have reported that patients hospitalized with COVID-19 have significantly increased coagulation activity with increasing D-dimer concentrations, and increased D-dimer levels are associated with severe disease/poor prognosis. ${ }^{25-27}$ In a study evaluating mortality in COVID-19, patients with both cerebrovascular disease and SARS-CoV-2 had higher D-dimer levels than patients with SARS-CoV-2 without cerebrovascular disease $(0.5 \mathrm{mg} / \mathrm{L}$ vs. $6.9 \mathrm{mg} / \mathrm{L}, \mathrm{p}<0.001) .{ }^{14}$ In our study, D-dimer levels were higher in the AIS group with COVID-19 (Table 3, Figure 2) and were associated with mortality. According to the ROC curve analysis, $630 \mathrm{ng} / \mathrm{mL}$ was determined as the best cut-off point for D-dimer. The sensitivity was $69.77 \%$ (95\% CI: 53.9 - 82.8) for individuals with D-dimer levels above $630 \mathrm{ng} / \mathrm{mL}$ and the specificity was $77.78 \%$ (95\% CI: 60.8-89.9) for those with $\leq 630 \mathrm{ng} / \mathrm{mL}$. In addition to D-dimer, $\mathrm{CRP}, \mathrm{LDH}$, ferritin, and troponin levels were also found to be significantly higher in the AIS group with COVID-19.

In a study evaluating the clinical characteristics and prognosis of patients with an acute respiratory distress syndrome (ARDS) or death with 201 definite COVID-19 diagnoses, a relationship was found between high ferritin levels and poor prognosis. ${ }^{28}$ In a meta-analysis, it was stated that the ferritin level was a risk factor in predicting disease severity and mortality in the course of COVI-19. ${ }^{29}$ In our study, the ferritin level was found to be significantly higher in the COVID-19-positive AIS group compared with the negative group, and it was found to be associated with mortality in the COVID-19-positive group. According to the ROC curve analysis, the best cut-off point for ferritin was $111 \mathrm{ng} / \mathrm{mL}$, the sensitivity was $83.72 \%$ and the specificity was $77.78 \%$.

In a multivariate analysis of a retrospective series of 440 patients with severe COVID-19, age, prolongation of prothrombin time, increased D-dimer, and thrombocytopenia were found to be associated with mortality. ${ }^{30}$ In our study, mortality was found to be associated with increased D-dimer levels, ferritin levels and thrombocytopenia in the AIS group with COVID-19 ( $\mathrm{p}=0.027, \mathrm{p}=0.034$, $\mathrm{p}=0.015$ ). With these findings, it can be thought that regular platelet counts and D-dimer level observations can be planned to monitor the level of coagulopathy and disease severity.

The single-center nature of this study has resulted in some limitations such as the limited number of patients, the retrospective analysis, not being able to perform advanced etiologic investigations in some patients with AIS and COVID-19, not including hemorrhagic strokes, and not being able to perform advanced laboratory 
investigations such as measuring antiphospholipid antibody levels and interleukin levels. The authors think that more data are needed in this area to determine the contribution of the processes involved in the pathogenesis of COVID-19 and stroke, and multicenter clinical studies with a higher number of patients are needed.

İn conclusion, patients with AIS and COVID-19 had a higher rate of intensive care need, higher intubation rates, longer hospital stay, higher mortality, and poorer prognosis than those with AIS without COVID-19. High levels of ferritin, D-dimer and fibrin degradation products were associated with poor prognosis. Further studies are needed to elucidate their role in the pathogenesis of AIS.

\section{DISCLOSURE}

Availability of data and material: The datasets used and/or analysed during the current study are available from the corresponding author upon reasonable request.

\section{Financial support: None}

Conflicts of interest: There were no conflicts of interest in this study.

\section{REFERENCES}

1. Romero-Sanchez CM, Diaz-Maroto I, Fernandez E, et al. Neurological manifestations in hospitalized patients with COVID-19: the ALBACOVID registry. Neurology 2020;95(8):e1060-e1070.

2. Avula A, Nalleballe K, Narula N, et al. COVID-19 presenting as stroke. Brain Behav Immun 2020 Jul;87:115-9.

3. Oxley TJ, Mocco J, Majidi S, et al. Large-Vessel Stroke as a Presenting Feature of Covid-19 in the Young. N Engl J Med 2020;382(20):e60.

4. Viguier A, Delamarre L, Duplantier J, Olivot JM, Bonneville F. Acute ischemic stroke complicating common carotid artery thrombosis during a severe COVID-19 infection.JNeuroradiol 2020;47(5):393-4.

5. Mao L, Jin H, Wang $\mathrm{M}$, et al. Neurologic manifestations of hospitalized patients with coronavirus disease 2019 in Wuhan, China. JAMA Neurol 2020;77:1-9.

6. Yaghi S, Ishida K, Torres J, et al. SARS-CoV-2 and stroke in a New York Healthcare System. Stroke 2020;51(7):2002-11.

7. Tan YK, Goh C, Leow AST, et al. COVID-19 and ischemic stroke: a systematic review and metasummary of the literature. J Thromb Thrombolysis 2020;50(3):587-95.

8. Panigada $\mathrm{M}$, Bottino $\mathrm{N}$, Tagliabue $\mathrm{P}$, et al. Hypercoagulability of COVID-19 patients in intensive care unit: A report of thromboelastography findings and other parameters of hemostasis. J Thromb
Haemost 2020;18(7):1738-42.

9. Valderrama EV, Humbert K, Lord A, Frontera J, Yaghi S. Severe Acute Respiratory Syndrome Coronavirus 2 Infection and Ischemic Stroke. Stroke 2020;51(7):e124-e127.

10. Adams HP Jr, Bendixen BH, Kappelle LJ, et al. Classification of subtype of acute ischemic stroke. Definitions for use in a multicenter clinical trial. TOAST. Trial of Org 10172 in Acute Stroke Treatment. Stroke 1993;24(1):35-41.

11. Bamford J, Sandercock P, Dennis M, Burn J, Warlow C. Classification and natural history of clinically identifiable subtypes of cerebral infarction. Lancet 1991;337(8756):1521-6.

12. Gunasekaran K, Amoah K, Rajasurya V, Buscher MG. Stroke in a young COVID-19 patient. QJM 2020;113(8):573-4.

13. Klein DE, Libman R, Kirsch C, Arora R. Cerebral venous thrombosis: A typical presentation of COVID-19 in the young. J Stroke Cerebrovasc Dis 2020;29(8):104989.

14. Li Y, Li M, Wang M, Zhou Y, Chang J, Xian Y, et al. Acute cerebrovascular disease following COVID-19: a single center, retrospective, observational study. Stroke Vasc Neurol. 2020 Sep;5(3):279-284. doi: 10.1136/svn-2020-000431

15. Zhao J, Li H, Kung D, Fisher M, Shen Y, Liu R. Impact of the COVID-19 epidemic on stroke care and potential solutions. Stroke 2020;51(7):1996-2001.

16. Montaner J, Barragán-Prieto A, Pérez-Sánchez S, et al. Break in the stroke chain of survival due to COVID-19. Stroke 2020;51(8):2307-14.

17. Lodigiani C, Iapichino G, Carenzo L, et al. Humanitas COVID-19 Task Force. Venous and arterial thromboembolic complications in COVID-19 patients admitted to an academic hospital in Milan, Italy. Thromb Res 2020;191:9-14.

18. Beyrouti R, Adams ME, Benjamin L, et al. Characteristics of ischaemic stroke associated with COVID-19. J Neurol Neurosurg Psychiatry 2020;91(8):889-91.

19. Zúñiga S, Cruz JL, Sola I, Mateos-Gómez PA, Palacio L, Enjuanes L. Coronavirus nucleocapsid protein facilitates template switching and is required for efficient transcription. J Virol 2010;84(4):2169-75.

20. Tang N, Bai H, Chen X, Gong J, Li D, Sun Z. Anticoagulant treatment is associated with decreased mortality in severe coronavirus disease 2019 patients with coagulopathy. J Thromb Haemost 2020;18(5):1094-99.

21. Connors JM, Levy JH. Thromboinflammation and the hypercoagulability of COVID-19. J Thromb Haemost 2020;18(7):1559-61.

22. Chen N, Zhou M, Dong X, et al. Epidemiological and clinical characteristics of 99 cases of 2019 novel coronavirus pneumonia in Wuhan, China: a descriptive study. Lancet 2020;395(10223):507-13.

23. Huang C, Wang Y, Li X, et al. Clinical features of patients infected with 2019 novel coronavirus in Wuhan, China. Lancet 2020;395(10223):497-506.

24. Benussi A, Pilotto A, Premi E, et al. Clinical characteristics and outcomes of inpatients with neurologic disease and COVID-19 in Brescia, Lombardy, Italy. Neurology 2020;95(7):e910-e920. 
25. Klok FA, Kruip MJHA, van der Meer NJM, et al. Incidence of thrombotic complications in critically ill ICU patients with COVID-19. Thromb Res 2020;191:145-7.

26. Guan WJ, Ni ZY, Hu Y, et al. Clinical characteristics of coronavirus disease 2019 in China. N Engl J Med 2020;382(18):1708-20.

27. Bowles L, Platton S, Yartey N, et al. Lupus anticoagulant and abnormal coagulation tests in patients with Covid-19. $N$ Engl J Med 2020;383(3):288-90.

28. Wu C, Chen X, Cai Y, et al. Risk factors associated with acute respiratory distress syndrome and death in patients with coronavirus disease 2019 pneumonia in Wuhan, China.JAMA Intern Med 2020;180(7):934-43.

29. Henry BM, de Oliveira MHS, Benoit S, Plebani M, Lippi G. Hematologic, biochemical and immune biomarker abnormalities associated with severe illness and mortality in coronavirus disease 2019 (COVID-19): a meta-analysis. Clin Chem Lab Med 2020;58(7):1021-8

30. Wang J, Hajizadeh $\mathrm{N}$, Moore EE, et al. Tissue plasminogen activator (tPA) treatment for COVID-19 associated acute respiratory distress syndrome (ARDS): A case series. J Thromb Haemost 2020;18(7):1752-5. 